\title{
Clustering Polysemic Subcategorization Frame Distributions Semantically
}

\author{
Anna Korhonen* \\ Computer Laboratory \\ University of Cambridge \\ 15 JJ Thomson Avenue \\ Cambridge CB3 OFD, UK \\ alk23@cl.cam.ac.uk
}

\author{
Yuval Krymolowski \\ Division of Informatics \\ University of Edinburgh \\ 2 Buccleuch Place \\ Edinburgh EH8 9LW \\ Scotland, UK \\ ykrymolo@inf.ed.ac.uk
}

\author{
Zvika Marx \\ Interdisciplinary Center \\ for Neural Computation, \\ The Hebrew University \\ Jerusalem, Israel \\ zvimecs.huji.ac.il
}

\begin{abstract}
Previous research has demonstrated the utility of clustering in inducing semantic verb classes from undisambiguated corpus data. We describe a new approach which involves clustering subcategorization frame $(\mathrm{SCF})$ distributions using the Information Bottleneck and nearest neighbour methods. In contrast to previous work, we particularly focus on clustering polysemic verbs. A novel evaluation scheme is proposed which accounts for the effect of polysemy on the clusters, offering us a good insight into the potential and limitations of semantically classifying undisambiguated SCF data.
\end{abstract}

\section{Introduction}

Classifications which aim to capture the close relation between the syntax and semantics of verbs have attracted a considerable research interest in both linguistics and computational linguistics (e.g. (Jackendoff, 1990; Levin, 1993; Pinker, 1989; Dang et al., 1998; Dorr, 1997; Merlo and Stevenson, 2001)). While such classifications may not provide a means for full semantic inferencing, they can capture generalizations over a range of linguistic properties, and can therefore be used as a means of reducing redundancy in the lexicon and for filling gaps in lexical knowledge.

This work was partly supported by UK EPSRC project GR/N36462/93: 'Robust Accurate Statistical Parsing (RASP)'.
Verb classifications have, in fact, been used to support many natural language processing (NLP) tasks, such as language generation, machine translation (Dorr, 1997), document classification (Klavans and Kan, 1998), word sense disambiguation (Dorr and Jones, 1996) and subcategorization acquisition (Korhonen, 2002).

One attractive property of these classifications is that they make it possible, to a certain extent, to infer the semantics of a verb on the basis of its syntactic behaviour. In recent years several attempts have been made to automatically induce semantic verb classes from (mainly) syntactic information in corpus data (Joanis, 2002; Merlo et al., 2002; Schulte im Walde and Brew, 2002).

In this paper, we focus on the particular task of classifying subcategorization frame ( $\mathrm{SCF}$ ) distributions in a semantically motivated manner. Previous research has demonstrated that clustering can be useful in inferring Levin-style semantic classes (Levin, 1993) from both English and German verb subcategorization information (Brew and Schulte im Walde, 2002; Schulte im Walde, 2000; Schulte im Walde and Brew, 2002).

We propose a novel approach, which involves: (i) obtaining SCF frequency information from a lexicon extracted automatically using the comprehensive system of Briscoe and Carroll (1997) and (ii) applying a clustering mechanism to this information. We use clustering methods that process raw distributional data directly, avoiding complex preprocessing steps required by many advanced methods (e.g. Brew and Schulte im Walde (2002)).

In contrast to earlier work, we give special empha- 
sis to polysemy. Earlier work has largely ignored this issue by assuming a single gold standard class for each verb (whether polysemic or not). The relatively good clustering results obtained suggest that many polysemic verbs do have some predominating sense in corpus data. However, this sense can vary across corpora (Roland et al., 2000), and assuming a single sense is inadequate for an important group of medium and high frequency verbs whose distribution of senses in balanced corpus data is flat rather than zipfian (Preiss and Korhonen, 2002).

To allow for sense variation, we introduce a new evaluation scheme against a polysemic gold standard. This helps to explain the results and offers a better insight into the potential and limitations of clustering undisambiguated SCF data semantically.

We discuss our gold standards and the choice of test verbs in section 2. Section 3 describes the method for subcategorization acquisition and section 4 presents the approach to clustering. Details of the experimental evaluation are supplied in section 5. Section 6 concludes with directions for future work.

\section{Semantic Verb Classes and Test Verbs}

Levin's taxonomy of verbs and their classes (Levin, 1993 ) is the largest syntactic-semantic verb classification in English, employed widely in evaluation of automatic classifications. It provides a classification of 3,024 verbs (4,186 senses) into 48 broad / 192 fine grained classes. Although it is quite extensive, it is not exhaustive. As it primarily concentrates on verbs taking NP and PP complements and does not provide a comprehensive set of senses for verbs, it is not suitable for evaluation of polysemic classifications.

We employed as a gold standard a substantially extended version of Levin's classification constructed by Korhonen (2003). This incorporates Levin's classes, 26 additional classes by Dorr (1997) ${ }^{1}$, and 57 new classes for verb types not covered comprehensively by Levin or Dorr.

110 test verbs were chosen from this gold standard, 78 polysemic and 32 monosemous ones. Some low frequency verbs were included to investigate the

\footnotetext{
${ }^{1}$ These classes are incorporated in the 'LCS database' (http://www.umiacs.umd.edu/ bonnie/verbs-English.lcs).
}

effect of sparse data on clustering performance. To ensure that our gold standard covers all (or most) senses of these verbs, we looked into WordNet (Miller, 1990) and assigned all the WordNet senses of the verbs to gold standard classes. ${ }^{2}$

Two versions of the gold standard were created: monosemous and polysemic. The monosemous one lists only a single sense for each test verb, that corresponding to its predominant (most frequent) sense in WordNet. The polysemic one provides a comprehensive list of senses for each verb. The test verbs and their classes are shown in table 1 . The classes are indicated by number codes from the classifications of Levin, Dorr (the classes starting with 0 ) and Korhonen (the classes starting with A). ${ }^{3}$ The predominant sense is indicated by bold font.

\section{Subcategorization Information}

We obtain our SCF data using the subcategorization acquisition system of Briscoe and Carroll (1997). We expect the use of this system to be beneficial: it employs a robust statistical parser (Briscoe and Carroll, 2002) which yields complete though shallow parses, and a comprehensive SCF classifier, which incorporates 163 SCF distinctions, a superset of those found in the ANLT (Boguraev et al., 1987) and COMLEX (Grishman et al., 1994) dictionaries. The SCFs abstract over specific lexicallygoverned particles and prepositions and specific predicate selectional preferences but include some derived semi-predictable bounded dependency constructions, such as particle and dative movement.

78 of these 'coarse-grained' SCFs appeared in our data. In addition, a set of 160 fine grained frames were employed. These were obtained by parameterizing two high frequency SCFs for prepositions: the simple PP and NP + PP frames. The scope was restricted to these two frames to prevent sparse data problems in clustering.

A SCF lexicon was acquired using this system from the British National Corpus (Leech, 1992, $\mathrm{BNC}$ ) so that the maximum of 7000 citations were

\footnotetext{
${ }^{2}$ As WordNet incorporates particularly fine grained sense distinctions, some senses were found which did not appear in our gold standard. As many of them appeared marginal and/or low in frequency, we did not consider these additional senses in our experiment.

${ }^{3}$ The gold standard assumes Levin's broad classes (e.g. class 10) instead of possible fine-grained ones (e.g. class 10.1).
} 


\begin{tabular}{|c|c|c|c|c|c|c|c|}
\hline $\begin{array}{l}\text { TEST } \\
\text { VERB }\end{array}$ & $\begin{array}{c}\text { GOLD STANDARD } \\
\text { CLASSES }\end{array}$ & $\begin{array}{l}\text { TEST } \\
\text { VERB }\end{array}$ & $\begin{array}{l}\text { GOLD STANDARD } \\
\text { CLASSES }\end{array}$ & $\begin{array}{l}\text { TEST } \\
\text { VERB }\end{array}$ & $\begin{array}{l}\text { GOLD STANDARD } \\
\text { CLASSES }\end{array}$ & $\begin{array}{l}\text { TEST } \\
\text { VERB }\end{array}$ & $\begin{array}{l}\text { GOLD STANDARD } \\
\text { CLASSES }\end{array}$ \\
\hline place & 9 & dye & $\mathbf{2 4 , 2 1 , 4 1}$ & focus & 31,45 & stare & 30 \\
\hline lay & 9 & build & 26,45 & force & 002,11 & glow & 43 \\
\hline drop & $\begin{array}{r}9,45,004,47 \\
51, \text { A } 54, \text { A } 30 \\
\end{array}$ & bake & $\mathbf{2 6}, 45$ & persuade & 002 & sparkle & 43 \\
\hline pour & $\mathbf{9}, 43,26,57,13,31$ & invent & 26,27 & urge & $\mathbf{0 0 2}, 37$ & $d r y$ & 45 \\
\hline load & 9 & publish & 26,25 & want & $\mathbf{0 0 2}, 005,29,32$ & shut & 45 \\
\hline settle & $\mathbf{9}, 46, \mathrm{~A} 16,36,55$ & cause & $\mathbf{2 7}, 002$ & need & $\mathbf{0 0 2}, 005,29,32$ & hang & $47,9,42,40$ \\
\hline fill & $\mathbf{9}, 45,47$ & generate & $\mathbf{2 7}, 13,26$ & grasp & 30,15 & sit & 47,9 \\
\hline remove & $\mathbf{1 0}, 11,42$ & induce & $\mathbf{2 7}, 002,26$ & understand & 30 & disappear & 48 \\
\hline withdraw & $\mathbf{1 0}, \mathrm{A} 30$ & acknowledge & 29, A25, A35 & conceive & 30, 29, A56 & vanish & 48 \\
\hline wipe & $\mathbf{1 0 , 9}$ & proclaim & $\mathbf{2 9}, 37, \mathrm{~A} 25$ & consider & 30,29 & march & 51 \\
\hline brush & $\mathbf{1 0}, 9,41,18$ & remember & $\mathbf{2 9}, 30$ & perceive & 30 & walk & 51 \\
\hline filter & 10 & imagine & $\mathbf{2 9}, 30$ & analyse & $\mathbf{3 4 , 3 5}$ & travel & 51 \\
\hline send & 11, A55 & specify & 29 & evaluate & $\mathbf{3 4 , 3 5}$ & hurry & $\mathbf{5 3}, 51$ \\
\hline ship & 11, A58 & establish & 29, A56 & explore & 35,34 & rush & $\mathbf{5 3}, 51$ \\
\hline transport & $\mathbf{1 1}, 31$ & suppose & 29,37 & investigate & 35,34 & begin & 55 \\
\hline carry & 11,54 & assume & 29, A35, A57 & agree & $\mathbf{3 6}, 22, \mathrm{~A} 42$ & continue & $\mathbf{5 5}, 47,51$ \\
\hline drag & $\mathbf{1 1}, 35,51,002$ & think & $\mathbf{2 9}, 005$ & communicate & 36,11 & snow & $\mathbf{5 7 , 0 0 2}$ \\
\hline push & $\mathbf{1 1}, 12,23,9,002$ & confirm & 29 & shout & 37 & rain & 57 \\
\hline pull & $\mathbf{1 1}, 12,13,23,40,016$ & believe & $\mathbf{2 9}, 31,33$ & whisper & 37 & $\sin$ & 003 \\
\hline give & 13 & admit & $\mathbf{2 9}, 024,045,37$ & talk & 37 & rebel & 003 \\
\hline lend & 13 & allow & $\mathbf{2 9}, 024,13,002$ & speak & 37 & risk & 008, A7 \\
\hline study & $\mathbf{1 4}, 30,34,35$ & act & 29 & say & 37,002 & gamble & $\mathbf{0 0 8}, 009$ \\
\hline hit & $\mathbf{1 8}, 17,47, \mathrm{~A} 56,31,42$ & behave & 29 & mention & 37 & beg & 015,32 \\
\hline bang & $\mathbf{1 8}, 43,9,47,36$ & feel & $\mathbf{3 0}, 31,35,29$ & eat & 39 & pray & 015,32 \\
\hline carve & $21,25,26$ & see & $\mathbf{3 0 , 2 9}$ & drink & 39 & seem & 020 \\
\hline add & $\mathbf{2 2}, 37, \mathrm{~A} 56$ & hear & 30, A32 & laugh & 40,37 & appear & $\mathbf{0 2 0}, 48,29$ \\
\hline $\operatorname{mix}$ & $\mathbf{2 2}, 26,36$ & notice & 30, A32 & smile & 40,37 & & \\
\hline colour & $\mathbf{2 4 , 3 1 , 4 5}$ & concentrate & 31,45 & look & 30,35 & & \\
\hline
\end{tabular}

Table 1: Test verbs and their monosemous/polysemic gold standard senses

used per test verb. The lexicon was evaluated against manually analysed corpus data after an empirically defined threshold of 0.025 was set on relative frequencies of SCFs to remove noisy SCFs. The method yielded $71.8 \%$ precision and $34.5 \%$ recall. When we removed the filtering threshold, and evaluated the noisy distribution, F-measure ${ }^{4}$ dropped from 44.9 to $38.51 .^{5}$

\section{Clustering Method}

Data clustering is a process which aims to partition a given set into subsets (clusters) of elements that are similar to one another, while ensuring that elements that are not similar are assigned to different clusters. We use clustering for partitioning a set of verbs. Our hypothesis is that information about SCFs and their associated frequencies is relevant for identifying semantically related verbs. Hence, we use SCFs as relevance features to guide the clustering process. ${ }^{6}$

\footnotetext{
${ }^{4} F=\frac{2 \cdot \text { precision } \cdot \text { recall }}{\text { precision }+ \text { recall }}$

${ }^{5}$ These figures are not particularly impressive because our evaluation is exceptionally hard. We use 1) highly polysemic test verbs, 2) a high number of SCFs and 3) evaluate against manually analysed data rather than dictionaries (the latter have high precision but low recall).

${ }^{6}$ The relevance of the features to the task is evident when comparing the probability of a randomly chosen pair of verbs $v e r b_{i}$ and $v e r b_{j}$ to share the same predominant sense $(4.5 \%)$ with the probability obtained when ver $b_{j}$ is the JS-divergence
}

We chose two clustering methods which do not involve task-oriented tuning (such as pre-fixed thresholds or restricted cluster sizes) and which approach data straightforwardly, in its distributional form: (i) a simple hard method that collects the nearest neighbours (NN) of each verb (figure 1), and (ii) the Information Bottleneck (IB), an iterative soft method (Tishby et al., 1999) based on information-theoretic grounds.

The NN method is very simple, but it has some disadvantages. It outputs only one clustering configuration, and therefore does not allow examination of different cluster granularities. It is also highly sensitive to noise. Few exceptional neighbourhood relations contradicting the typical trends in the data are enough to cause the formation of a single cluster which encompasses all elements.

Therefore we employed the more sophisticated IB method as well. The IB quantifies the relevance information of a SCF distribution with respect to output clusters, through their mutual information $I$ (Clusters; SCFs). The relevance information is maximized, while the compression information I(Clusters; Verbs) is minimized. This ensures optimal compression of data through clusters. The tradeoff between the two constraints is realized

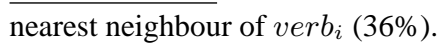




\section{NN Clustering:}

1. For each verb $v$ :

2. Calculate the JS divergence between the SCF distributions of $v$ and all other verbs:

$$
\operatorname{JS}(p, q)=\frac{1}{2}\left[D\left(p \| \frac{p+q}{2}\right)+D\left(q \| \frac{p+q}{2}\right)\right]
$$

3. Connect $v$ with the most similar verb;

4. Find all the connected components

Figure 1: Connected components nearest neighbour (NN) clustering. $D$ is the Kullback-Leibler distance.

through minimizing the cost term:

$$
\mathcal{L}=I(\text { Clusters } ; \text { Verbs })-\beta I(\text { Cluster } ; \text { SCFs }),
$$

where $\beta$ is a parameter that balances the constraints.

The IB iterative algorithm finds a local minimum of the above cost term. It takes three inputs: (i) $\mathrm{SCF}-$ verb distributions, (ii) the desired number of clusters $\mathcal{K}$, and (iii) the value of $\beta$.

Starting from a random configuration, the algorithm repeatedly calculates, for each cluster $K$, verb $V$ and SCF $S$, the following probabilities: (i) the marginal proportion of the cluster $p(K)$; (ii) the probability $p(S \mid K)$ for a SCF to occur with members of the cluster; and (iii) the probability $p(K \mid V)$ for a verb to be assigned to the cluster. These probabilities are used, each in its turn, for calculating the other probabilities (figure 2). The collection of all $p(S \mid K)$ 's for a fixed cluster $K$ can be regarded as a probabilistic center (centroid) of that cluster in the SCF space.

The IB method gives an indication of the most informative values of $\mathcal{K}{ }^{7}$ Intensifying the weight $\beta$ attached to the relevance information $I$ (Clusters; $\mathrm{SCF} s)$ allows us to increase the number $\mathcal{K}$ of distinct clusters being produced (while too small $\beta$ would cause some of the output clusters to be identical to one another). Hence, the relevance information grows with $\mathcal{K}$. Accordingly, we consider as the most informative output configurations those for which the relevance information increases more sharply between $\mathcal{K}-1$ and $\mathcal{K}$ clusters than between $\mathcal{K}$ and $\mathcal{K}+1$.

\footnotetext{
${ }^{7}$ Most works on clustering ignore this issue and refer to an arbitrarily chosen number of clusters, or to the number of gold standard classes, which cannot be assumed in realistic applications.
}

\section{IB Clustering (fixed $\beta$ ):}

Perform till convergence, for each time step

$t=1,2, \ldots$ :

1. $z_{t}(K, V)=p_{t-1}(K) e^{-\beta D\left[p(S \mid V) \| p_{t-1}(S \mid K)\right.}$

(When $t=1$, initialize $z_{t}(K, V)$ arbitrarily)

2. $p_{t}(K \mid V)=\frac{z_{t}(K, V)}{\sum_{K^{\prime}} z_{t}\left(K^{\prime}, V\right)}$

3. $p_{t}(K)=\sum_{V} p(V) p_{t}(K \mid V)$

4. $p_{t}(S \mid K)=\sum_{V} p(S \mid V) p_{t}(V \mid K)$

Figure 2: Information Bottleneck (IB) iterative clustering. $D$ is the Kullback-Leibler distance.

When the weight of relevance grows, the assignment to clusters is more constrained and $p(K \mid V)$ becomes more similar to hard clustering. Let

$$
K(V)=\underset{K}{\operatorname{argmax}} p(K \mid V)
$$

denote the most probable cluster of a verb $V$. For $\mathcal{K} \geq 30$, more than $85 \%$ of the verbs have $p(K(V) \mid V)>90 \%$ which makes the output clustering approximately hard. For this reason, we decided to use only $K(V)$ as output and defer a further exploration of the soft output to future work.

\section{Experimental Evaluation}

\subsection{Data}

The input data to clustering was obtained from the automatically acquired SCF lexicon for our 110 test verbs (section 2). The counts were extracted from unfiltered (noisy) SCF distributions in this lexicon. ${ }^{8}$ The NN algorithm produced 24 clusters on this input. From the IB algorithm, we requested $\mathcal{K}=2$ to 60 clusters. The upper limit was chosen so as to slightly exceed the case when the average cluster size $110 / \mathcal{K}=2$. We chose for evaluation the IB results for $\mathcal{K}=25,35$ and 42 . For these values, the SCF relevance satisfies our criterion for a notable improvement in cluster quality (section 4). The value $\mathcal{K}=35$ is very close to the actual number (34) of predominant senses in the gold standard. In this way, the IB yields structural information beyond clustering.

\footnotetext{
${ }^{8}$ This yielded better results, which might indicate that the unfiltered "noisy" SCFs contain information which is valuable for the task.
} 


\subsection{Method}

A number of different strategies have been proposed for evaluation of clustering. We concentrate here on those which deliver a numerical value which is easy to interpret, and do not introduce biases towards specific numbers of classes or class sizes. As we currently assign a single sense to each polysemic verb (sec. 5.4) the measures we use are also applicable for evaluation against a polysemous gold standard.

Our first measure, the adjusted pairwise precision (APP), evaluates clusters in terms of verb pairs (Schulte im Walde and Brew, 2002) ${ }^{9}$ :

$$
\mathrm{APP}=\frac{1}{\mathcal{K}} \sum_{i=1}^{\mathcal{K}} \frac{\text { num. of correct pairs in } k_{i}}{\text { num. of pairs in } k_{i}} \cdot \frac{\left|k_{i}\right|-1}{\left|k_{i}\right|+1} .
$$

APP is the average proportion of all within-cluster pairs that are correctly co-assigned. It is multiplied by a factor that increases with cluster size. This factor compensates for a bias towards small clusters.

Our second measure is derived from purity, a global measure which evaluates the mean precision of the clusters, weighted according to the cluster size (Stevenson and Joanis, 2003). We associate with each cluster its most prevalent semantic class, and denote the number of verbs in a cluster $K$ that take its prevalent class by $n_{\text {prevalent }}(K)$. Verbs that do not take this class are considered as errors. Given our task, we are only interested in classes which contain two or more verbs. We therefore disregard those clusters where $n_{\text {prevalent }}(K)=1$. This leads us to define modified purity:

$$
m \text { PUR }=\frac{\sum_{\text {prevalent }\left(k_{i}\right) \geq 2} n_{\text {prevalent }}\left(k_{i}\right)}{\text { number of verbs }} .
$$

The modification we introduce to purity removes the bias towards the trivial configuration comprised of only singletons.

\subsection{Evaluation Against the Predominant Sense}

We first evaluated the clusters against the predominant sense, i.e. using the monosemous gold standard. The results, shown in Table 2, demonstrate that both clustering methods perform significantly

\footnotetext{
${ }^{9}$ Our definition differs by a factor of 2 from that of Schulte im Walde and Brew (2002).
}

\begin{tabular}{|c|c|c|c||c|c|}
\hline Alg. & $\mathcal{K}$ & +PP & - PP & +PP & - PP \\
\hline & \multicolumn{3}{|c|}{ APP: } & \multicolumn{2}{|c|}{ mPUR: } \\
\hline NN & $(24)$ & $21 \%$ & $19 \%$ & $48 \%$ & $45 \%$ \\
\hline & 25 & $12 \%$ & $9 \%$ & $39 \%$ & $32 \%$ \\
IB & 35 & $14 \%$ & $9 \%$ & $48 \%$ & $38 \%$ \\
& 42 & $15 \%$ & $9 \%$ & $50 \%$ & $39 \%$ \\
\hline RAND & 25 & \multicolumn{2}{|c||}{$3 \%$} & \multicolumn{2}{c|}{$15 \%$} \\
\hline
\end{tabular}

Table 2: Clustering performance on the predominant senses, with and without prepositions. The last entry presents the performance of random clustering with $\mathcal{K}=25$, which yielded the best results among the three values $\mathcal{K}=25,35$ and 42 .

better on the task than our random clustering baseline. Both methods show clearly better performance with fine-grained SCFs (with prepositions, +PP) than with coarse-grained ones (-PP).

Surprisingly, the simple NN method performs very similarly to the more sophisticated IB. Being based on pairwise similarities, it shows better performance than IB on the pairwise measure. The IB is, however, slightly better according to the global measure $(2 \%$ with $\mathcal{K}=42)$. The fact that the NN method performs better than the IB with similar $\mathcal{K}$ values (NN $\mathcal{K}=24$ vs. IB $\mathcal{K}=25$ ) seems to suggest that the JS divergence provides a better model for the predominant class than the compression model of the IB. However, it is likely that the IB performance suffered due to our choice of test data. As the method is global, it performs better when the target classes are represented by a high number of verbs. In our experiment, many semantic classes were represented by two verbs only (section 2).

Nevertheless, the IB method has the clear advantage that it allows for more clusters to be produced. At best it classified half of the verbs correctly according to their predominant sense ( $m$ PUR $=50 \%)$. Although this leaves room for improvement, the result compares favourably to previously published results ${ }^{10}$. We argue, however, that evaluation against a monosemous gold standard reveals only part of the picture.

\footnotetext{
${ }^{10}$ Due to differences in task definition and experimental setup, a direct comparison with earlier results is impossible. For example, Stevenson and Joanis (2003) report an accuracy of $29 \%$ (which implies $m$ PUR $\leq 29 \%$ ), but their task involves classifying 841 verbs to 14 classes based on differences in the predicate-argument structure.
} 


\begin{tabular}{|c|c|c||c|c|}
\hline $\mathcal{K}$ & $\begin{array}{c}\text { Pred. } \\
\text { sense }\end{array}$ & $\begin{array}{c}\text { Multiple } \\
\text { senses }\end{array}$ & $\begin{array}{c}\text { Pred. } \\
\text { sense }\end{array}$ & $\begin{array}{c}\text { Multiple } \\
\text { senses }\end{array}$ \\
\hline & APP: & \multicolumn{2}{|c|}{ mPUR: } \\
\hline NN: & $21 \%$ & $29 \%(23 \%+5 \sigma)$ & $48 \%$ & $60 \%(46 \%+2 \sigma)$ \\
$(24)$ & $21 \%$ & $18 \%(14 \%+5 \sigma)$ & $39 \%$ & $48 \%(43 \%+3 \sigma)$ \\
\hline IB: & $12 \%$ & $18 \%$ & $47 \%$ & $59 \%(50 \%+4 \sigma)$ \\
25 & $14 \%$ & $20 \%(16 \%+6 \sigma)$ & $50 \%$ & $59 \%(54 \%+2 \sigma)$ \\
35 & $14 \%(16 \%+3 \sigma)$ & $50 \%$ & $19 \%(16 \%)$ \\
42 & $15 \%$ & \multicolumn{3}{|c}{} \\
\hline
\end{tabular}

Table 3: Evaluation against the monosemous (Pred.) and polysemous (Multiple) gold standards. The figures in parentheses are results of evaluation on randomly polysemous data + significance of the actual figure. Results were obtained with finegrained SCFs (including prepositions).

\subsection{Evaluation Against Multiple Senses}

In evaluation against the polysemic gold standard, we assume that a verb which is polysemous in our corpus data may appear in a cluster with verbs that share any of its senses. In order to evaluate the clusters against polysemous data, we assigned each polysemic verb $V$ a single sense: the one it shares with the highest number of verbs in the cluster $K(V)$.

Table 3 shows the results against polysemic and monosemous gold standards. The former are noticeably better than the latter (e.g. IB with $\mathcal{K}=42$ is $9 \%$ better). Clearly, allowing for multiple gold standard classes makes it easier to obtain better results with evaluation.

In order to show that polysemy makes a nontrivial contribution in shaping the clusters, we measured the improvement that can be due to pure chance by creating randomly polysemous gold standards. We constructed 100 sets of random gold standards. In each iteration, the verbs kept their original predominant senses, but the set of additional senses was taken entirely from another verb - chosen at random. By doing so, we preserved the dominant sense of each verb, the total frequency of all senses and the correlations between the additional senses.

The results included in table 3 indicate, with $99.5 \%$ confidence ( $3 \sigma$ and above), that the improvement obtained with the polysemous gold standard is not artificial (except in two cases with $95 \%$ confidence).

\subsection{Qualitative Analysis of Polysemy}

We performed qualitative analysis to further investigate the effect of polysemy on clustering perfor-

\begin{tabular}{|c|r|c|}
\hline $\begin{array}{c}\text { Different } \\
\text { Senses }\end{array}$ & Pairs & $\begin{array}{c}\text { Fraction } \\
\text { in cluster }\end{array}$ \\
\hline 0 & 39 & $51 \%$ \\
1 & 85 & $10 \%$ \\
2 & 625 & $7 \%$ \\
3 & 1284 & $3 \%$ \\
4 & 1437 & $3 \%$ \\
\hline
\end{tabular}

Table 4: The fraction of verb pairs clustered together, as a function of the number of different senses between pair members (results of the NN algorithm)

\begin{tabular}{|c|r|c|r|c|}
\hline Common & \multicolumn{2}{|c|}{ one irregular } & \multicolumn{2}{c|}{ no irregular } \\
\cline { 2 - 5 } Senses & Pairs & in cluster & Pairs & in cluster \\
\hline 0 & 2180 & $3 \%$ & 3018 & $3 \%$ \\
1 & 388 & $9 \%$ & 331 & $12 \%$ \\
2 & 44 & $20 \%$ & 31 & $35 \%$ \\
\hline
\end{tabular}

Table 5: The fraction of verb pairs clustered together, as a function of the number of shared senses (results of the NN algorithm)

mance. The results in table 4 demonstrate that the more two verbs differ in their senses, the lower their chance of ending up in the same cluster. From the figures in table 5 we see that the probability of two verbs to appear in the same cluster increases with the number of senses they share. Interestingly, it is not only the degree of polysemy which influences the results, but also the type. For verb pairs where at least one of the members displays 'irregular' polysemy (i.e. it does not share its full set of senses with any other verb), the probability of co-occurrence in the same cluster is far lower than for verbs which are polysemic in a 'regular' manner (Table 5).

Manual cluster analysis against the polysemic gold standard revealed a yet more comprehensive picture. Consider the following clusters (the IB output with $\mathcal{K}=42$ ):

A1: talk (37), speak (37)

A2: $\operatorname{look}(30,35)$, stare (30)

A3: focus $(31,45)$, concentrate $(31,45)$

A4: $\operatorname{add}(22,37, \mathrm{~A} 56)$

We identified a close relation between the clustering performance and the following patterns of semantic behaviour:

1) Monosemy: We had 32 monosemous test verbs. 10 gold standard classes included 2 or more or these. 7 classes were correctly acquired using clustering (e.g. A1), indicating that clustering monosemous verbs is fairly 'easy'. 
2) Predominant sense: 10 clusters were examined by hand whose members got correctly classified together, despite one of them being polysemous (e.g. A2). In 8 cases there was a clear indication in the data (when examining SCFs and the selectional preferences on argument heads) that the polysemous verb indeed had its predominant sense in the relevant class and that the co-occurrence was not due to noise.

3) Regular Polysemy: Several clusters were produced which represent linguistically plausible intersective classes (e.g. A3) (Dang et al., 1998) rather than single classes.

4) Irregular Polysemy: Verbs with irregular polysemy ${ }^{11}$ were frequently assigned to singleton clusters. For example, add (A4) has a 'combining and attaching' sense in class 22 which involves NP and PP SCFs and another 'communication' sense in 37 which takes sentential SCFs. Irregular polysemy was not a marginal phenomenon: it explains 5 of the 10 singletons in our data.

These observations confirm that evaluation against a polysemic gold standard is necessary in order to fully explain the results from clustering.

\subsection{Qualitative Analysis of Errors}

Finally, to provide feedback for further development of our verb classification approach, we performed a qualitative analysis of errors not resulting from polysemy. Consider the following clusters (the IB output for $\mathcal{K}=42$ ):

B1: place (9), build $(26,45)$, publish $(26,25)$, carve $(21,25,26)$

B2: $\sin (003)$, rain $(57)$, snow $(57,002)$

B3: agree (36, 22, A42), appear $(020,48,29)$, begin $(55)$, continue $(55,47,51)$

B4: $\operatorname{beg}(015,32)$

Three main error types were identified:

1) Syntactic idiosyncracy: This was the most frequent error type, exemplified in B1, where place is incorrectly clustered with build, publish and carve merely because it takes similar prepositions to these verbs (e.g. in, on, into).

2) Sparse data: Many of the low frequency verbs (we had 12 with frequency less than 300) performed

\footnotetext{
${ }^{11}$ Recall our definition of irregular polysemy, section 5.4.
}

poorly. In B2, sin (which had 53 occurrences) is classified with rain and snow because it does not occur in our data with the preposition against the 'hallmark' of its gold standard class ('Conspire Verbs').

3) Problems in SCF acquisition: These were not numerous but occurred e.g. when the system could not distinguish between different control (e.g. subject/object equi/raising) constructions (B3).

\section{Discussion and Conclusions}

This paper has presented a novel approach to automatic semantic classification of verbs. This involved applying the NN and IB methods to cluster polysemic SCF distributions extracted from corpus data using Briscoe and Carroll's (1997) system. A principled evaluation scheme was introduced which enabled us to investigate the effect of polysemy on the resulting classification.

Our investigation revealed that polysemy has a considerable impact on the clusters formed: polysemic verbs with a clear predominant sense and those with similar regular polysemy are frequently classified together. Homonymic verbs or verbs with strong irregular polysemy tend to resist any classification.

While it is clear that evaluation should account for these cases rather than ignore them, the issue of polysemy is related to another, bigger issue: the potential and limitations of clustering in inducing semantic information from polysemic SCF data. Our results show that it is unrealistic to expect that the 'important' (high frequency) verbs in language fall into classes corresponding to single senses. However, they also suggest that clustering can be used for novel, previously unexplored purposes: to detect from corpus data general patterns of semantic behaviour (monosemy, predominant sense, regular/irregular polysemy).

In the future, we plan to investigate the use of soft clustering (without hardening the output) and develop methods for evaluating the soft output against polysemous gold standards. We also plan to work on improving the accuracy of subcategorization acquisition, investigating the role of noise (irregular / regular) in clustering, examining whether different syntactic/semantic verb types require different ap- 
proaches in clustering, developing our gold standard classification further, and extending our experiments to a larger number of verbs and verb classes.

\section{References}

B. Boguraev, E. J. Briscoe, J. Carroll, D. Carter, and C. Grover. 1987. The derivation of a grammaticallyindexed lexicon from the longman dictionary of contemporary english. In Proc. of the $25^{\text {th }} A C L$, pages 193-200, Stanford, CA.

C. Brew and S. Schulte im Walde. 2002. Spectral clustering for german verbs. In Conference on Empirical Methods in Natural Language Processing, Philadelphia, USA.

E. J. Briscoe and J. Carroll. 1997. Automatic extraction of subcategorization from corpora. In $5^{\text {th }} A C L$ Conference on Applied Natural Language Processing, pages 356-363, Washington DC.

E. J. Briscoe and J. Carroll. 2002. Robust accurate statistical annotation of general text. In $3^{\text {rd }}$ International Conference on Language Resources and Evaluation, pages 1499-1504, Las Palmas, Gran Canaria.

H. T. Dang, K. Kipper, M. Palmer, and J. Rosenzweig. 1998. Investigating regular sense extensions based on intersective Levin classes. In Proc. of COLING/ACL, pages 293-299, Montreal, Canada.

B. Dorr and D. Jones. 1996. Role of word sense disambiguation in lexical acquisition: predicting semantics from syntactic cues. In $16^{\text {th }}$ International Conference on Computational Linguistics, pages 322-333, Copenhagen, Denmark.

B. Dorr. 1997. Large-scale dictionary construction for foreign language tutoring and interlingual machine translation. Machine Translation, 12(4):271-325.

R. Grishman, C. Macleod, and A. Meyers. 1994. Comlex syntax: building a computational lexicon. In International Conference on Computational Linguistics, pages 268-272, Kyoto, Japan.

R. Jackendoff. 1990. Semantic Structures. MIT Press, Cambridge, Massachusetts.

E. Joanis. 2002. Automatic verb classification using a general feature space. Master's thesis, University of Toronto.

J. L. Klavans and M. Kan. 1998. Role of verbs in document analysis. In Proc. of COLING/ACL, pages 680686, Montreal, Canada.

A. Korhonen. 2002. Subcategorization Acquisition. Ph.D. thesis, University of Cambridge, UK.
A. Korhonen. 2003. Extending Levin's Classification with New Verb Classes. Unpublished manuscript, University of Cambridge Computer Laboratory.

G. Leech. 1992. 100 million words of english: the british national corpus. Language Research, 28(1):1-13.

B. Levin. 1993. English Verb Classes and Alternations. Chicago University Press, Chicago.

P. Merlo and S. Stevenson. 2001. Automatic verb classification based on statistical distributions of argument structure. Computational Linguistics, 27(3):373-408.

P. Merlo, S. Stevenson, V. Tsang, and G. Allaria. 2002. A multilingual paradigm for automatic verb classification. In Proc. of the $40^{\text {th }} A C L$, Pennsylvania, USA.

G. A. Miller. 1990. WordNet: An on-line lexical database. International Journal of Lexicography, 3(4):235-312.

S. Pinker. 1989. Learnability and Cognition: The Acquisition of Argument Structure. MIT Press, Cambridge, Massachusetts.

J. Preiss and A. Korhonen. 2002. Improving subcategorization acquisition with WSD. In ACL Workshop on Word Sense Disambiguation: Recent Successes and Future Directions, Philadelphia, USA.

D. Roland, D. Jurafsky, L. Menn, S. Gahl, E. Elder, and C. Riddoch. 2000. Verb subcatecorization frequency differences between business-news and balanced corpora. In ACL Workshop on Comparing Corpora, pages 28-34.

S. Schulte im Walde and C. Brew. 2002. Inducing german semantic verb classes from purely syntactic subcategorisation information. In Proc. of the $40^{\text {th }} A C L$, Philadephia, USA.

S. Schulte im Walde. 2000. Clustering verbs semantically according to their alternation behaviour. In Proc. of COLING-2000, pages 747-753, Saarbrücken, Germany.

S. Stevenson and E. Joanis. 2003. Semi-supervised verb-class discovery using noisy features. In Proc. of CoNLL-2003, Edmonton, Canada.

N. Tishby, F. C. Pereira, and W. Bialek. 1999. The information bottleneck method. In Proc. of the $37^{\text {th }}$ Annual Allerton Conference on Communication, Control and Computing, pages 368-377. 